\title{
A METRIC INEQUALITY CHARACTERIZING BARYCENTERS AND OTHER PETTIS INTEGRALS
}

\author{
RUSSELL G. BILYEU
}

\begin{abstract}
Certain Pettis integrals, including barycenters of probability measures on weakly compact subsets of Banach spaces, are characterized by an integral inequality which refers only to distances between points, avoiding any reference to the linear structure of the Banach space. This is an elaboration of the Mazur-Ulam discovery that the metric determines the linear structure.
\end{abstract}

This paper extends from finite sums to fairly general integrals the following theorem of Wolfe [7]:

THEOREM. If $\left\|x-x_{0}\right\| \leqslant \sum a_{i}\left\|x-x_{i}\right\|\left(a_{i} \geqslant 0, \sum a_{i}=1\right)$ for each $x$ in $a$ Banach space then $x_{0}=\sum a_{i} x_{i}$.

Wolfe's ingenious technique for avoiding the tedious argument of [3] made use of the derivative of the norm. This technique adapts to the present case because of a result of Asplund [2] insuring the density of smooth points in weakly compactly generated Banach spaces. The most direct extension of Wolfe's theorem is the following.

THEOREM 1. Suppose that $S$ is a weakly compact subset of the Banach space $X$ and that $\mu$ is a regular Borel probability measure on the weak Borel subsets of $S$. Then the barycenter

$$
x_{0}=\int_{S} x d \mu(x)
$$

exists (as a Bochner integral) and is the unique element $x_{0} \in X$ satisfying

$$
\left\|y-x_{0}\right\| \leqslant \int_{S}\|y-x\| d \mu(x)
$$

for all $y \in X$.

This result is a corollary to more general results concerning Pettis integrals of certain functions with values in $X$, to which we now turn our attention.

The one-sided derivative of the norm may be defined as

$$
D^{+} \cdot(x, y)=\lim (\|n x+y\|-\|n x\|), \quad n \rightarrow \infty .
$$

Received by the editors April 5, 1977 and, in revised form, July 12, 1977.

AMS (MOS) subject classifications (1970). Primary 46G05, 46G10; Secondary 52A40.

Key words and phrases. Banach, barycenter, Pettis integral.

(C) American Mathematical Society 1978 
If $D^{+}(x,-y)=-D^{+}(x, y)$ for each $y \in X$ then $x$ is called a smooth point of $X$ and $D^{+}(x, y)$ is written $D(x, y)$. In that case, $D(x, \cdot)$ is a continuous linear functional on $X$. Such functionals are called Gateaux differentials of the norm.

$(S, \Sigma, \mu)$ denotes a measure space with $\mu \geqslant 0$ and $\mu(S)=1$, and $f: S \rightarrow X$ denotes a function such that, for each $x \in X,\|x-f()\|$ is $\mu$-integrable.

THEOREM 2. If $\left\|x-x_{0}\right\| \leqslant \int\|x-f()\| d \mu$ for each $x \in X$ then, for each $u \in X$,

$$
D^{+}\left(u, x_{0}\right) \leqslant \int D^{+}(u, f()) d \mu .
$$

Furthermore, for each smooth point $u$ of $X$,

$$
D\left(u, x_{0}\right)=\int D(u, f()) d \mu .
$$

Proof. Observe that the sequence of functions $\|n u+f()\|-\|n u\|, n=$ $1,2,3, \ldots$, is nonincreasing and that each function in the sequence has its absolute value bounded by $\|f()\|$. The dominated convergence theorem implies that

$$
\begin{aligned}
D^{+}\left(u, x_{0}\right) & =\lim \left(\left\|n u+x_{0}\right\|-\|n u\|\right) \\
& \leqslant \lim \int(\|n u+f()\|-\|n u\|) d \mu \\
& =\int \lim (\|n u+f()\|-\|n u\|) d u \\
& =\int D^{+}(u, f()) d \mu .
\end{aligned}
$$

If $u$ is a smooth point we also conclude that

$$
\begin{aligned}
-D\left(u, x_{0}\right) & =D^{+}\left(u,-x_{0}\right)=D^{+}\left(-u, x_{0}\right) \leqslant \int D^{+}(-u, f()) d \mu \\
& =\int D^{+}(u,-f()) d \mu=-\int D^{+}(u, f()) d \mu ;
\end{aligned}
$$

therefore, $D\left(u, x_{0}\right)=\int D(u, f()) d \mu$.

TheOREM 3. Suppose $\left\|x-x_{0}\right\| \leqslant \int\|x-f()\| d \mu$ for each $x \in X$ and the Pettis integral $\int f d \mu$ exists in $X$. Suppose also that the Gateaux differentials of the norm are total over $X$. Then $x_{0}=\int f d \mu$.

Proof. By Theorem 2, if $u$ is a smooth point then

$$
D\left(u, x_{0}\right)=\int D(u, f()) d \mu .
$$

The Pettis integral is so defined that

$$
D\left(u, \int f d \mu\right)=\int D(u, f()) d \mu .
$$


Thus $x_{0}-\int f d \mu$ is annihilated by $D(u, \cdot)$, and the totality hypothesis implies $x_{0}=\int f d \mu$.

LEMMA. The Gateaux differentials of the norm are total over a weakly compactly generated Banach space.

Proof. Let $X$ be a weakly compactly generated Banach space. Suppose that $y \in X$ is annihilated by every Gateaux differential of the norm. Let $x$ be a smooth point of $X$. For each positive integer $n$,

$$
\|x\|=\|n x+x\|-\|n x\| \leqslant\|n x+y\|+\|y-x\|-\|n x\| .
$$

Consequently, $\|x\| \leqslant\|y-x\|+D(x, y)$. But $D(x, y)=0$; hence $\|x\| \leqslant \| y$ $-x \|$ for each smooth point $x$. By a theorem of Asplund [2], the smooth points are dense in $X$. Considering a sequence of smooth points converging to $y$, we conclude that $y=0$.

Theorem 4. Suppose $\left\|x-x_{0}\right\| \leqslant \int\|x-f()\| d \mu$ for each $x \in X$, the Pettis integral $\int f d \mu$ exists in $X$, and the range of $f$ is contained in a weakly compact set. Then $x_{0}=\int f d \mu$.

Proof. Let $Y$ be a closed linear subspace of $X$ generated by a weakly compact convex set which contains the range of $f$. By an argument which may be found in the book of Alfsen [1, Proposition 1.2.1], the Pettis integral $\int f d \mu$ belongs to $Y$. As a consequence of the Lemma, Theorem 3 applies.

THEOREM 5. Suppose $\left\|x-x_{0}\right\| \leqslant \int\|x-f()\| d \mu$ for each $x \in X$ and the Gateaux differentials of the norm are fundamental (span a dense set) in $X^{*}$. Then the Pettis integral $\int f d \mu$ exists and is $x_{0}$.

Proof. Pick $x^{*} \in X^{*}$. The equation

$$
x^{*}\left(x_{0}\right)=\int x^{*} \circ f d \mu
$$

holds in case $x^{*}$ is a Gateaux differential of the norm, as a consequence of Theorem 2. By linearity, the same equation holds if $x^{*}$ is a linear combination of Gateaux differentials of the norm. In general, there exists a sequence $\left(x_{n}^{*}\right)$ such that $x_{n}^{*} \rightarrow x^{*}$ and

$$
x_{n}^{*}\left(x_{0}\right)=\int x_{n}^{*} \circ f d \mu .
$$

The dominated convergence theorem implies

$$
x^{*}\left(x_{0}\right)=\lim \int x_{n}^{*} \circ f d \mu=\int x^{*} \circ f d \mu .
$$

Proof of Theorem 1. It is known that the Bochner integral $\int x d \mu(x)$ exists. Being unable to supply a reference, we supply a proof. Grothendieck proved that $\mu$ has separable support [5]. Therefore the range of the identity function is $\mu$-essentially separable. Since the identity function is weakly measurable, it follows from fundamental results of Pettis [6] and Bochner [4] that the Bochner integral $\int x d \mu(x)$ exists. Call it $x_{0}$. The desired inequality is 
then a consequence of basic properties of integrals. Uniqueness follows from Theorem 4.

REMARK. The requirement that Gateaux differentials be total over $X$ is satisfied by reflexive spaces and by separable spaces, these being weakly compactly generated. The requirement that the Gateaux differentials be fundamental in $X^{*}$ is satisfied by reflexive spaces and also by $c_{0}(A)$ for an arbitrary set $A$. It is not satisfied by the separable Banach space $C[0,1]$, since the linear span of the point masses is not dense in the dual. Whether such requirements are needed is unknown-we have no example of a Pettis integrable function for which there exist more than one $x_{0}$ satisfying the metric inequality.

\section{REFERENCES}

1. E. M. Alfsen, Compact convex sets and boundary integrals, Springer-Verlag, New York and Berlin, 1971.

2. E. Asplund, Fréchet differentiability of convex functions, Acta Math. 121 (1968), 31-48.

3. R. G. Bilyeu, Metric definition of the linear structure, Proc. Amer. Math. Soc. 25 (1970), 205-206.

4. S. Bochner, Integration von Funktionen deren Wert die Elemente eines Vektorraumes sind, Fund. Math. 20 (1933), 262-276.

5. A. Grothendieck, Sur les applications linéaires faiblement compactes d'espaces du type $C(K)$, Canad. J. Math. 5 (1953), 129-173.

6. B. J. Pettis, On integration in vector spaces, Trans. Amer. Math. Soc. 44 (1938), 277-304.

7. D. Wolfe, Metric inequalities and convexity, Proc. Amer. Math. Soc. 40 (1973), 559-562.

Department of Mathematics, North Texas State University, Denton, TeXas 76203 